\title{
Three-dimensional printing in medicine: Opportunities for development of optimal CT scanning protocols
}

\author{
Zhonghua Sun, Sultan Aldosari
}

Discipline of Medical Radiation Sciences, School of Molecular and Life Sciences, Curtin University, Perth, Western Australia, 6845, Australia

\section{REVIEW}

Please cite this paper as: Sun Z, Aldosari S. Three-dimensional printing in medicine: Opportunities for development of optimal CT scanning protocols. AMJ 2018;11(11):529-532. https://doi.org/10.21767/AMJ.2018.3533

\section{Corresponding Author:}

Prof Zhonghua Sun

Discipline of Medical Radiation Sciences, School of Molecular and Life Sciences, Curtin University, Perth, WA, 6845, Australia Email: z.sun@curtin.edu.au

\section{ABSTRACT}

Three-dimensional (3D) printing has shown great promise with increased applications in the medical field. 3D printed models are reported to accurately replicate normal anatomy and pathology, assist pre-surgical planning and simulation, serve as a useful tool for medical education and improve patient-doctor communication. Another unique application of 3D printed realistic models is to develop optimal computed tomography (CT) scanning protocols for investigation of scanning parameters with low radiation dose while still achieving diagnostic images. This editorial highlights the application of using patient-specific 3D printed pulmonary artery model for investigation of optimal CT pulmonary angiography protocols for detection of pulmonary embolism.

\section{Key Words}

Computed tomography pulmonary angiography, image quality, model, pulmonary embolism, simulation, threedimensional printing

\section{Implications for Practice:}

\section{What is known about this subject?}

$3 \mathrm{D}$ printing is increasingly used in many clinical applications ranging from orthopaedics to cardiovascular and cerebrovascular disease.

\section{What new information is offered in this review?}

Use of 3D printed physical models for investigation of optimal CT scanning protocols has not been conducted previously. This review summarises our experience of testing different CT pulmonary angiography protocols on a patient-specific 3D printed pulmonary artery model with simulation of thrombus in the main pulmonary arteries.

3. What are the implications for research, policy, or practice?

3D printed model allows for studying optimal CT scanning protocols with the aim of identifying technical parameters with lowest radiation dose but acceptable image quality for the diagnosis of pulmonary embolism.

\section{Introduction}

Three-dimensional (3D) printing is a fast evolving technique with increased applications in the medical field. $^{1-5}$ It is generally agreed that 3D printed models based on medical imaging data, most commonly from computed tomography (CT) or magnetic resonance imaging (MRI) accurately replicate normal anatomy and pathology, with better visualisation of 3D relationship between anatomical structures and complex pathologies. 3D printed models are originally applied in orthopaedic and orthodontic fields, ${ }^{6}$ and have now been extended to assist pre-surgical planning and simulation of surgical procedures, serve as a useful tool for education of medical students and improve patient-doctor communication. $^{1-9}$

In addition to the above-mentioned applications, 3D printed physical models can be used for testing different CT scanning protocols with the aim of identifying optimal scanning parameters with lowest radiation dose while still achieving diagnostic image quality. This is confirmed by our recent studies showing the feasibility of this 
approach. This editorial provides a summary of the new application of 3D printed models for development of optimal CT scanning protocols.

\section{Quantitative assessment of CT pulmonary angiography protocols based on 3D printed model}

In our first report, we have successfully created a 3D printed pulmonary artery model based on a selected case with normal CT pulmonary angiography (CTPA) images. ${ }^{10}$ The 3D model consists of pulmonary trunk, main pulmonary arteries and side branches with normal diameters. Figure 1 shows the image post-processing and segmentation steps to generate 3D printed model. The model was printed with elastoplastic material with property similar to the normal arterial wall. Measurements of anatomical locations of pulmonary arteries showed very high accuracy among the original CT images, the standard tessellation language (STL) file and the CT images of the model, indicating high precision of the model in replicating anatomical structures. ${ }^{10}$

Following the first report, we conducted further experiments by simulating thrombus in the main pulmonary arteries and tested different CTPA protocols on a 128-slice dual source CT scanner. ${ }^{11}$ In this recent study, a range of $\mathrm{kVp}$ including 70,80 , 100 and 120 and various pitch values comprising 0.9, 2.2 and 3.2 were used to determine the effect of different protocols on image quality and radiation dose. Animal blood clots that were obtained from a local butcher were used to simulate thrombus in the pulmonary arteries. Image quality was determined by measuring signal-to-noise ratio (SNR) within the thrombus and in the main pulmonary arteries (Figure 2). Results showed no significant differences in the SNR measurements across all 100 and $120 \mathrm{kVp}$ protocols $(p>0.05)$, whether low pitch of 0.9 or high pitch of 3.2 protocol was used, although SNR was higher in the $120 \mathrm{kVp}$ protocols. SNR was found to be significantly lower in the 70 and $80 \mathrm{kVp}$ with high-pitch 3.2 protocols when compared to that measured in other protocols using pitch values of 0.9 and $2.2(p<0.05)$. This is observed in these measurements within the thrombus and in the main pulmonary arteries. Figure 3 shows increased image noise in the low $\mathrm{kVp}$ and high-pitch protocols, especially apparent in the $70 \mathrm{kVp}$ protocol, although thrombus is still visible in these pulmonary arteries. 3D virtual intravascular endoscopy (VIE) images demonstrate the effect of different CTPA protocols on the visualisation of intraluminal thrombus and pulmonary arterial wall with CTPA protocol of $70 \mathrm{kVp}$ and high pitch 3.2 resulting in suboptimal visualisation of thrombus and arterial wall (Figure 4). This study further validates the feasibility of using low-dose CTPA protocol (80 and $100 \mathrm{kVp}$ ) in the detection of pulmonary embolism with dose reduction up to 80 per cent. However, lowering $k V p$ to
70 and use of high pitch 3.2 raises concerns due to image quality being compromised because of increased image noise. Increasing pitch is associated with decreasing spatial resolution, especially in the z-axis resolution, thus, resulting in suboptimal image quality in the $70 \mathrm{kVp}$ protocols. This can be addressed by increasing the mAs.

CTPA is currently the first line imaging technique in the diagnosis of suspected pulmonary embolism with high diagnostic yield and low radiation dose. ${ }^{12-16}$ High-pitch CTPA is more attractive due to its associated very low radiation dose without affecting image quality and reduction of motion artefacts in cardiorespiratory imaging due to short scanning times and elimination of breathhold, and this is confirmed by some recent reports. ${ }^{13-16}$ Testing different CT scanning protocols on phantoms represents a common approach, while utilising patientspecific 3D printed models for investigation of optimal imaging parameters has not been reported in the literature. Abdullah et al. developed a 3D printed cardiac insert phantom with filling materials having similar CT attenuations to those of patient's CT images. ${ }^{17}$ Despite uniqueness of this cardiac phantom, authors did not test different scanning protocols on the 3D printed model. In contrast, our phantom experiments further advanced 3D printing to more practical applications in medical imaging field for investigation of optimal CT protocols. Results of our study will pay the way for encouraging more studies with use of 3D printed models for developing optimal CT scanning protocols.

\section{Summary and conclusion}

Patient-specific 3D printed models of normal anatomy and pathology are playing an important role in the current clinical practice, with applications of 3D printed models contributing to better patient care and management. 3D printed realistic models can also be used for testing different CT scanning protocols for determining the optimal ones with lowest radiation dose and acceptable image quality. Our recent study has demonstrated the feasibility of using 3D printed pulmonary model for developing optimal CT pulmonary angiography protocols. Potential limitations of this 3D printed model will need to be overcome in further studies, such as placing the 3D printed model in a similar anatomical background consisting of lungs, heart, ribs, and spine, etc., thus allowing robust conclusions to be drawn. Further, investigation of optimal CT pulmonary angiography protocols for detection of peripheral pulmonary embolism with both qualitative and quantitative assessments is desirable. 


\section{References}

1. Olivieri $\sqcup$, Krieger $A$, Loke $\mathrm{YH}$, et al. Three-dimensional printing of intracardiac defects from three-dimensional echocardiographic images: feasibility and relative accuracy. J Am Sco Echocardiogr. 2015;28: 392-97.

2. Cantinotti M, Valverde I, Kutty S. Three-dimensional printed models in congenital heart disease. Int J Cardiovasc Imaging. 2017;33(1):137-144.

3. Valverde I, Gomez-Ciriza G, Hussain T, et al. Three dimensional printed models for surgical planning of complex congenital heart defects: an international multicenter study. Eur J Cardiothorac Surg. 2017;52(6):1139-1148.

4. Lau I, Liu D, Xu L, et al. Clinical value of patient-specific three-dimensional printing of congenital heart disease: Quantitative and qualitative Assessments. PLoS One. 2018;13:e0194333.

5. Sun Z, Liu D. A systematic review of clinical value of threedimensional printing in renal disease. Quant Imaging Med Surg. 2018;8:311-325.

6. Zanetti EM, Aldieri $A$, Terzini $M$, et al. Additive manufacturing custom load-bearing implantable devices. AMJ. 2017;10:694-670.

7. Giannopoulos AA, Steigner ML, George $E$, et al. Cardiothoracic applications of 3-dimensional printing. J Thorac Imaging. 2016;31:253-272.

8. Zanetti EM, Bignardi C. Mock-up in hip arthroplasty preoperative planning. Acta Bioeng Biomech. 2013;15:123128.

9. Speranza D, Citro D, Padula F, et al. Additive manufacturing techniques for the reconstruction of $3 \mathrm{D}$ fetal faces. Appl Bionics Biomech. 2017;2017:9701762.

10. Aldosari S, Squelch A, Sun Z. Patient-specific 3D printed pulmonary artery model: A preliminary study. Digit Med. 2017;3:170-177.

11. Aldosari S, Jansen S, Sun Z. Optimization of computed tomography pulmonary angiography protocols using 3D printed model with simulation of pulmonary embolism. Quant Imaging Med Surg. 2018.

12. Sun Z, Lei J. Diagnostic yield of CT pulmonary angiography in the diagnosis of pulmonary embolism: a single center experience. Interv Cardiol 2017;9:191-198.

13. Lu GM, Luo S, Meinel FG, et al. High-pitch computed tomography pulmonary angiography with iterative reconstruction at $80 \mathrm{kVp}$ and $20 \mathrm{~mL}$ contrast agent volume. Eur Radiol. 2014;24:3260-3268.

14. Sabel BO, Buric K, Karara N, et al. High-pitch CT pulmonary angiography in third generation dual-source CT: image quality in an unselected patient population. PLOS ONE. 2016;11:e0146949.

15. Boos J, Kropil P, Lanzman RS, et al. CT pulmonary angiography: simultaneous low-pitch dual-source acquisition mode with $70 \mathrm{kVp}$ and $40 \mathrm{ml}$ of contrast medium and comparison with high-pitch spiral dualsource acquisition with automated tube potential selection. Br J Radiol. 2016;89:20151059.

16. Bucher AM, Kerl MJ, Albrecht MH, et al. Systematic comparison of reduced tube current protocols for high-pitch and standard-pitch pulmonary CT angiography in a large single-center population. Acad Radiol. 2016;23:619-627.

17. Abdullah KA, McEntee MF, Reed $W$, et al. Development of an organ-specific insert phantom generated using a 3D printer for investigations of cardiac computed tomography protocols. J Med Radiat Sci. 2018;65:175-183.

\section{PEER REVIEW}

Peer reviewed.

\section{CONFLICTS OF INTEREST}

The authors declare that they have no competing interests.

\section{FUNDING}

None

Figure 1: Flow diagram shows the image post-processing and segmentation processes from original 2D CT images to creation of 3D printed model

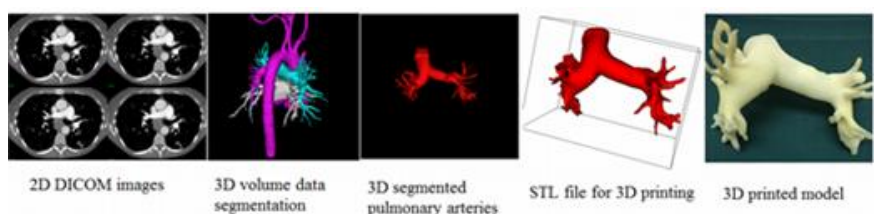

Original DICOM images were used to create 3D volume rendering image for displaying contrast-enhanced vessels (blue colour-pulmonary arteries, pink colour-aorta and its branches, white colour-left atrium and pulmonary veins). 3D volume rendering of pulmonary artery tree is segmented through a semi-automatic segmentation approach with manual editing. STL (Standard Tessellation Language) file of 3D segmented volume data was generated for 3D printing of patient-specific 3D printed model. Reprint with permission under the open access from Aldosari S, Squelch A and Sun Z. ${ }^{10}$ 
Figure 2: Measurement of signal-to-noise ratio (SNR)

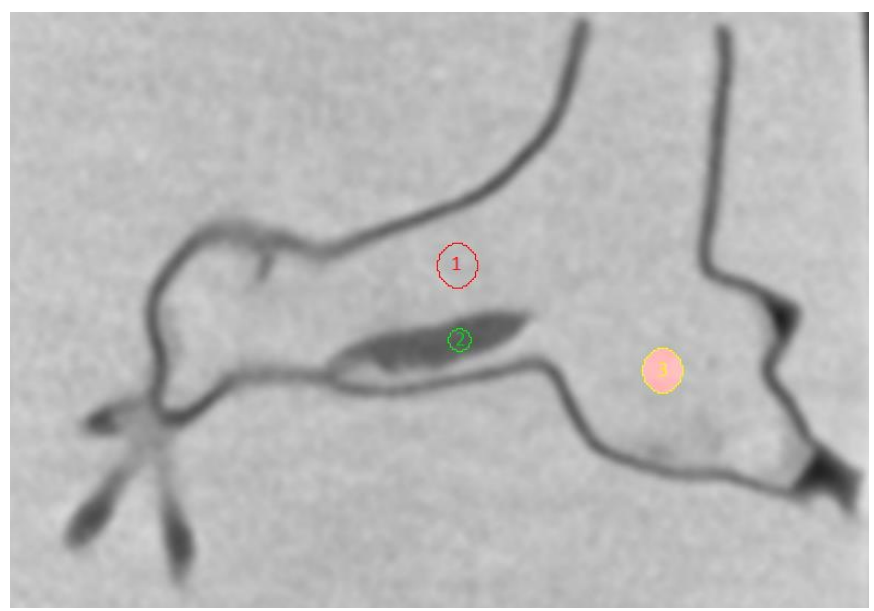

SNR was measured in the left and right main pulmonary arteries as shown in the large regions of interest (ROI). The small ROI indicates SNR measurement in the thrombus.

Figure 3: Computed tomography pulmonary angiography protocols with use of different $\mathrm{kVp}$ (70 and 80 ) and pitch values $(0.9,2.2$ and 3.2$)$. The corresponding $m A s$ for these protocols are as follows: 60, 125 and $126 \mathrm{mAs}$ for the $70 \mathrm{kVp}$ with different pitch protocols, 38, 98 and $146 \mathrm{mAs}$ for the 80 $k V p$ with different pitch protocols

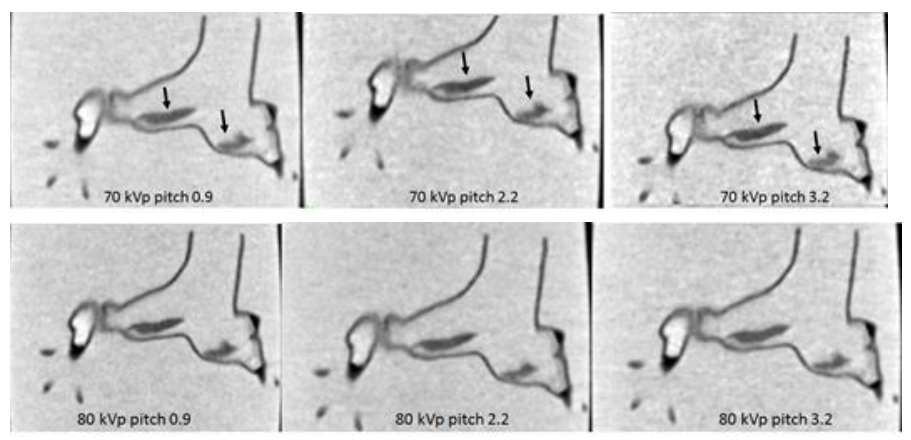

Image noise was increased with 70 and $80 \mathrm{kVp}$ with high pitch of 3.2 protocols. This is especially apparent with $70 \mathrm{kVp}$ protocol. In contrast, image quality remained the same with 100 and $120 \mathrm{kVp}$ protocols, regardless of pitch values (not shown). Arrows refer to the thrombus in the main pulmonary arteries. Volume CT dose index (CTDIvol) was $0.41,0.15$ and $0.12,0.42,0.21$ and $0.21 \mathrm{mGy}$ for 70 and $80 \mathrm{kVp}$ with different pitch values, respectively. Reprint with permission under the open access from Aldosari S, Jansen S and Sun Z. ${ }^{11}$
Figure 4: Virtual intravascular endoscopy (VIE) views of thrombus in images acquired with different computed tomography pulmonary angiography (CTPA) protocols
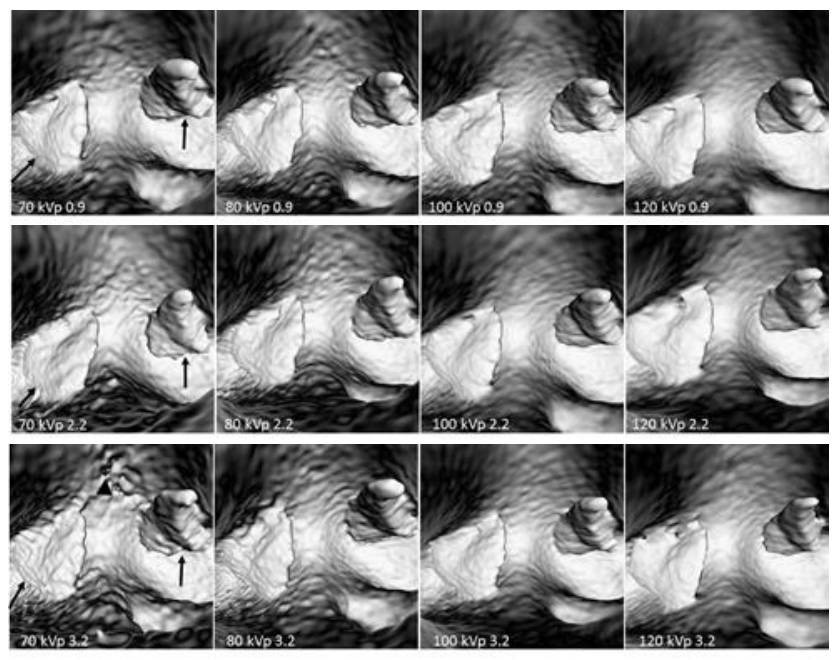

Intraluminal views of the thrombus are clearly demonstrated with CTPA protocols using 70, 80, 100 and $120 \mathrm{kVp}$ and pitch values of 0.9 and 2.2. When high pitch of 3.2 was used, irregular appearance of the thrombus (arrows) and some artefacts (arrowhead) appeared in the low kVp 70 protocol when compared to other protocols. Reprint with permission under the open access from Aldosari S, Jansen S and Sun Z. ${ }^{11}$ 